\title{
TEMPERATURE DEPENDENCE OF THE ENERGY GAP IN $\mathrm{Cd}_{1-x} \mathrm{Fe}_{x} \mathrm{Se}$
}

\author{
J.A. GAJ ${ }^{a}$, NguYen The Khol ${ }^{* a}$, M. ARCiszewskA ${ }^{b}$ AND K. LebeCKI ${ }^{a}$ \\ ${ }^{a}$ Institute of Experimental Physics, Warsaw University \\ Hoża 69, 00-681 Warszawa, Poland \\ ${ }^{b}$ Institute of Physics, Polish Academy of Sciences \\ Al. Lotników 32/46, 02-668 Warszawa, Poland
}

\begin{abstract}
The energy gap and magnetic susceptibility of $\mathrm{Cd}_{0.85} \mathrm{Fe}_{0.15}$ Se were measured in function of temperature. Experiments showed that the magnetic contribution to the variation of the energy gap in $\mathrm{Cd}_{1-x} \mathrm{Fe}_{x} \mathrm{Se}$ is not proportional to the product of magnetic susceptibility and temperature as it has been observed in $\mathrm{Mn}^{++}$-containing semiconductors.
\end{abstract}

PACS numbers: 75.50.Pp

\section{Introduction}

It has been shown [1] that magnetic contribution to the energy gap of $\mathrm{Mn}^{++}$-containing semimagnetic semiconductors is negative and proportional to the product of magnetic susceptibility and temperature:

$$
\Delta E_{\mathbf{g}}^{\operatorname{mag}}=b T \chi(x, T),
$$

where $b$ is related to wave-vector dependence of ion-carrier exchange integral $J_{q}$. Within a simple cutoff model:

$$
b=-J_{0}^{2} \frac{m_{\mathrm{h}} j(j+1) k_{\mathrm{B}}}{\hbar^{2} \pi^{2} N_{0} N_{\mathrm{A}}\left(g \mu_{\mathrm{B}}\right)^{2}} q_{\mathrm{c}},
$$

where $J_{0}$ is ion-carrier exchange constant at $q=0, q_{\mathrm{c}}$ is its cutoff value, $j=3 / 2$ is the hole momentum and $m_{\mathrm{h}}$ its a verage effective mass, $N_{0}$ denotes number of unit cells per unit volume, $N_{\mathrm{A}}$ is Avogadro's number, $g$ - gyromagnetic factor and $\mu_{\mathrm{B}}$ - Bohr magneton.

\footnotetext{
*On leave from Pedagogical University, Hanoi
} 
As pointed out by J. Blinowski and P. Kacman [2] the approximations producing the above result may not be adequate in case of magnetic systems possessing internal excitations of order of $k_{\mathrm{B}} T$. This is the case of $\mathrm{Fe}^{++}$in CdSe. The purpose of this work is to examine the validity of the approach of Ref. [1] in the case of iron-containing semimagnetic semiconductor $\mathrm{Cd}_{1-x} \mathrm{Fe}_{x} \mathrm{Se}$. This material is the only Fe-containing semimagnetic semiconductor where relatively high concentration of magnetic ions $(x=0.15)$ is available. Furthermore, analogous results obtained on its Mn-based counterpart CdMnSe have been published by L. Bryja and J.A. Gaj [3].

\section{Experiment and discussion}

Measurements of absorption in the free exciton region were performed on $\mathrm{Cd}_{1-x} \mathrm{Fe}_{x} \mathrm{Se}$ and CdSe samples obtained from Bridgman-grown single crystals by local etching to a thickness below $1 \mu \mathrm{m}$. Figure 1 shows values of energy gap (free exciton absorption peak) plotted for $x=0$ (CdSe) and $x=0.15$ as a function of temperature. To check the validity of Eq. (1), magnetic susceptibility measure-

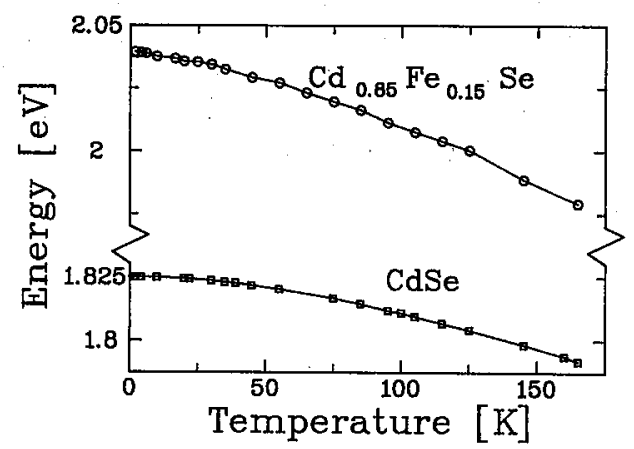

Fig. 1. Free exciton absorption peak energy plotted vs. temperature for CdSe and $\mathrm{Cd}_{0.85} \mathrm{Fe}_{0.15} \mathrm{Se}$.

ments were also performed on a sample cut out of the same crystal. Figure $2 \mathrm{~b}$ shows the results, plotted in the form $\chi T$ vs. $T$, suitable for our purpose. The difference:

$$
\left[E_{\mathrm{g}}(x, T)-E_{\mathrm{g}}(x, 0)\right]-\left[E_{\mathrm{g}}(0, T)-E_{\mathrm{g}}(0,0)\right]
$$

represented in Fig. 2a is rather superlinear as a function of $T$, whereas $\chi T$ (Fig. 2b) has a completely different (sublinear) form. This confirms the conclusions of Ref. [2] and shows a need for a detailed theoretical calculation corresponding to the experimental results.

If we try to compare the values of the two curves in the high-temperature limit (where we can hope that the model of Ref. [1] can still be used as a rough approximation) we obtain $b=-0.078 \mathrm{eV} \cdot \mathrm{Gs}^{2} \cdot \mathrm{erg}^{-2} \cdot \mathrm{K}^{-2}$. Using available data 


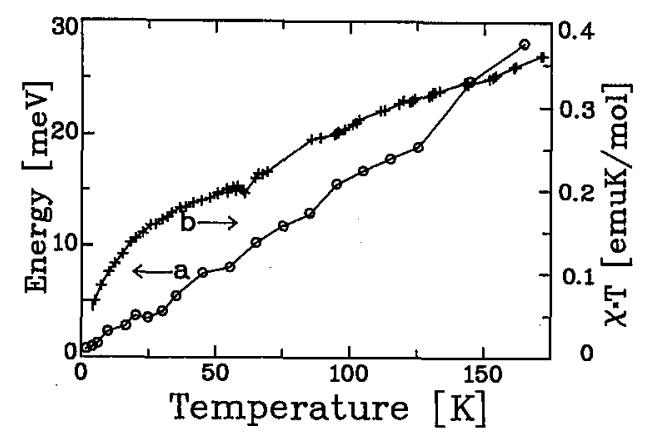

Fig. 2. Difference between temperature variation of the energy gap of $\mathrm{Cd}_{0.85} \mathrm{Fe}_{0.15} \mathrm{Se}$ and that of CdSe (a) and product of magnetic susceptibility and temperature plotted versus temperature for $\mathrm{Cd}_{0.85} \mathrm{Fe}_{0.15} \mathrm{Se}(\mathrm{b})$.

on valence band effective mass $m_{\mathrm{h}}=0.7 m_{0}[3]$ and ion-hole exchange integral at $q=0, N_{0} \beta=-1.53 \mathrm{eV}$ [4], we obtain from Eq. (2) $q_{\mathrm{c}}=2.56 \times 10^{7} \mathrm{~cm}^{-1}$.

These values can be compared to the results obtained by L. Bryja and J.A. Gaj [3] for $\mathrm{Cd}_{1-x} \mathrm{Mn}_{x} \mathrm{Se}: b=-0.085 \mathrm{eV} \cdot \mathrm{Gs}^{2} \cdot \mathrm{erg}^{-2} \cdot \mathrm{K}^{-2}$ and $q_{\mathrm{c}}=3.77 \times$ $10^{7} \mathrm{~cm}^{-1}$.

The smaller value of $q_{\mathrm{c}}$ for $\mathrm{Fe}^{++}$corresponds to an intuitive idea of bigger effective size of the $\mathrm{Fe}^{++}$ion compared to the $\mathrm{Mn}^{++}$one.

\section{Acknowledgment}

We thank Prof. A. Mycielski for the samples used in this work.

\section{References}

[1] J.A. Gaj, A. Golnik, Acta Phys. Pol. A71, 197 (1987).

[2] J. Blinowski, P. Kacman, Acta Phys. Pol. A75, 215 (1989).

[3] L. Bryja, J.A. Gaj, Acta Phys. Pol. A73, 459 (1988).

[4] D. Scalbert, M. Guillot, A. Mauger, J.A. Gaj, J. Cernogora, C. Benoit à la Guillaume, A. Mycielski, Solid State Commun. 76, 977 (1990). 